\title{
High performance fibre-optic acoustic sensor array using a distributed EDFA and hybrid TDM/DWDM, scalable to 4096 sensors
}

\author{
Yi Liao ${ }^{* a}$, Ed Austin ${ }^{\mathrm{b}}$, Philip J. Nash ${ }^{\mathrm{b}}$, Stuart A. Kingsley ${ }^{\mathrm{b}}$, David J. Richardson ${ }^{\mathrm{a}}$ \\ ${ }^{a}$ Optoelectronics Research Centre, University of Southampton, UK; \\ ${ }^{\mathrm{b}}$ Stingray Geophysical Limited, Surrey KT6 6AP, UK
}

\begin{abstract}
An amplified 16 channel dense wavelength division multiplexing (DWDM) array architecture is presented for interferometric fibre optic sensor array systems. This architecture employs a distributed Erbium doped fibre amplifier (EDFA) scheme to decrease the array insertion loss, and employs time division multiplexing (TDM) at each wavelength to increase the number of sensors that can be supported. The first experimental demonstration of this system is reported including results which show the potential for multiplexing and interrogating up to 4096 sensors using a single telemetry fibre pair with good system performance.
\end{abstract}

Keywords: Distributed amplified array, fibre sensor array, acoustic sensing, EDFA, multiplexing, phase resolution

\section{INTRODUCTION}

Interferometric fiber optic acoustic sensors based on measuring the phase modulation of light travelling in an optical fiber due to the strains developed on the fibre by a measurand, have been researched for nearly four decades. This research has been driven by their use in a number of important practical applications, for example military sonar and seismic sensing. Even with the ultra-high sensitivities available, single channel applications are only appropriate in a few instances for cost reasons. Fortunately, fibre-optic sensors lend themselves to multiplexing, which allows the cost of the lasers and other expensive components to be divided between many channels. Many multiplexing schemes have been proposed and investigated based on techniques including time, frequency, coherence, and wavelength multiplexing, and combinations thereof [1-5]. In each instance splitting/recombination loss ultimately limits the scalability of the approach, with the number of fibres required for telemetry a further critical factor that significantly impacts the overall system cost and practicality. The largest interferometric fibre-optic sensor array reported to date consisted of a time- and wavelengthdivision multiplexed architecture combining up to 256 sensors onto a single fibre pair [6].

In this paper, to our knowledge, we report the first experimental demonstration of acoustic measurements in an amplified TDM/DWDM array architecture. The system is capable of interrogating up to 4096 sensors through only one interlink fibre pair whilst satisfying the demanding performance levels required by many applications.

\section{PRINCIPLE OF OPERATION}

The principle of the array topology proposed here is based on loss compensation of the distribution bus using a distributed multi-section EDFA in a hybrid TDM/DWDM architecture. The array is addressed via two fibre buses, the distribution bus (top) and the return bus (bottom), as illustrated in Figure 1. The optical pulses from the wavelength multiplexed sources are coupled into the distribution bus. At the first optical drop multiplexer (ODM), the signal at wavelength $\lambda 1$ is coupled into the first TDM group (all sensors driven by one wavelength), passes through the constituent sub-array of TDM sensors, and is then coupled onto the return bus through the optical add multiplexer (OAM), which delivers it to the detector. The remaining wavelengths of the input interrogation signal continue along the distribution bus to the subsequent ODMs, successively feeding into each TDM group, before being multiplexed onto the return bus. This architecture performs the 'add' and 'drop' functions by separate devices and thus provides the advantage that if the telemetry fibre is severed within the array then only the signals from the TDM groups corresponding to ODM/OAMs positioned after the break are lost. EDFA segments are distributed along the return bus to compensate the insertion loss, and the gain of each amplifying section is set to exactly compensate for the subsequent losses for each sensor group. The 
amplifiers are all co-pumped remotely through the existing return bus fibre by $1480 \mathrm{~nm}$ pump lasers located at the front and/or back ends of the array. The couplers are ODM/OAMs so that the pump never couples out of the bus. Since each amplifier must compensate for only a small insertion loss, (except for amp0), they provide a low gain and require just a small pump power. The input pump power is selected to be much larger than the first amplifier's saturation pump power. Therefore this amplifier absorbs only a small fraction of the pump power so that the large remaining power is transmitted to the downstream amplifiers, which also operate in a pump saturation regime.

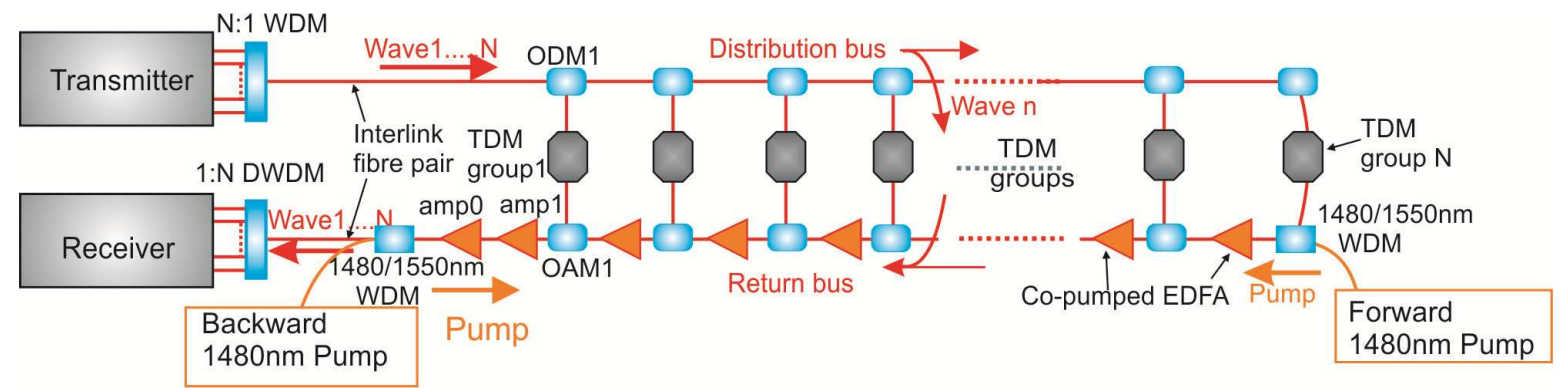

Figure 1 Proposed amplified TDM /DWDM array topology

This array architecture provides dramatic advantages for large scale arrays. The topology uses TDM within amplified DWDM architectures for the first time, such that the number of multiplexed sensors is now given by the product of the number of TDM sensors and the number of wavelengths, which is increased by several times compared to the number multiplexed in amplified TDM arrays with the same interrogation repetition rate [4]. The interrogation repetition rate determines the bandwidth available for the phase modulated signal to occupy, and it is dependent on the number of sensors and the length of fibre per sensor. The array allows the interrogation of thousands of multiplexed sensors utilizing only one single telemetry fibre pair, which significantly decreases the array complexity, cost, weight, and provides for ready expansion. The insertion loss of the array is compensated by the distributed amplification, which leaves a higher power budget for the remote transmission of the signal along the cable, and limits the chance of nonlinear effects within the fibre bus. These benefits are of prime importance in many applications.

\section{EXPERIMENTAL SETUP}

Although a larger number of wavelengths can be multiplexed, due to cost, practicality and equipment availability we use 16 wavelengths in our proof-of-principle experimental configuration, as shown in Figure 2. This sensor system comprises three principal components: a transmitter, the amplified array architecture and a receiver section. The transmitter consists of four narrow linewidth $(\sim 10 \mathrm{kHz})$ fibre lasers for interrogation, and a further twelve DFB lasers (from a DWDM telecom source bank) to provide suitable signals to assess the optical amplification performance of the array (the linewidth of the DFB lasers was too broad to allow them to be used for sensor interrogation). The four narrow linewidth sources were multiplexed, pulsed, frequently-shifted, and amplified, then launched into a "TDM group". The output signal was then combined with the twelve CW sources and then launched into the amplified array architecture. The maximum launch power into the array was $22 \mathrm{dBm}$ per wavelength, limited by nonlinear effects.

The experimental arrangement was constructed with only one "TDM group" representing all of the sensors in the array. This was located before the array so that each wavelength suffered the loss of this device. This should provide similar optical performance in terms of loss to a fully-loaded system in which a TDM group is included between each ODM/OAM pair and is obviously far more convenient from a practical perspective. The "TDM group" itself comprised a single fibre Michelson interferometer sensor along with a tunable attenuator to simulate a prescribed number of additional "missing sensors" as described below. To simulate an applied acoustic signal, one arm of the interferometer is wrapped around a piezoelectric transducer (PZT), allowing the sensor to be modulated with an ac voltage.

The amplified array comprises 15 pairs of ODM/OAMs along two fibre buses to support the 16 wavelengths. The insertion losses of the ODM/OAMs were measured to determine the lengths of the Er-doped fibre required in each amplifier to compensate their loss. An amplifier is located after each ODM/OAM pair. In our experiments all amplifiers are co-pumped by a $1466 \mathrm{~nm}$ pump source coupled into the return bus with a maximum output pump power of $400 \mathrm{~mW}$. The lengths of amplifier segments used throughout the network were determined by detailed numerical simulations of the system supplemented by detailed amplifier measurements which allowed us to determine the salient physical properties of the erbium doped fibre used. 


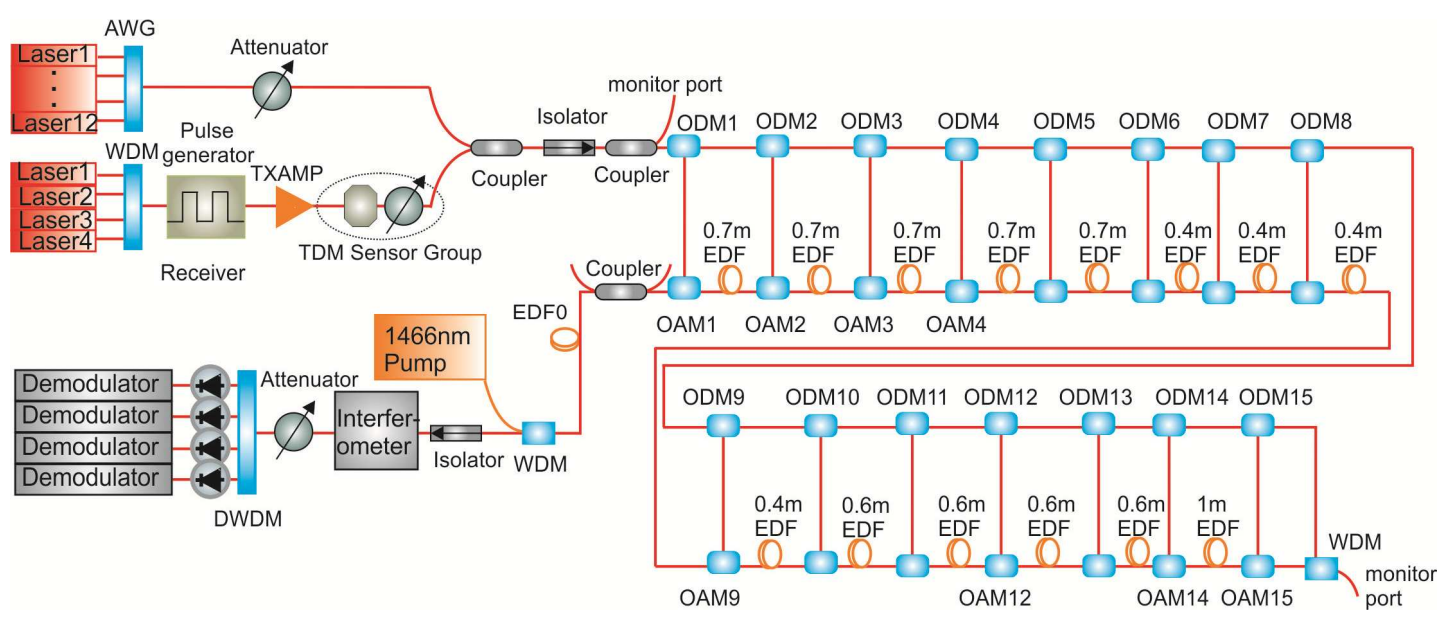

Figure 2 Example experimental setup of the 16 wavelength architecture for phase noise interrogation from 4 narrow linewidth fibre lasers and representing 12 DFB lasers

The return signals from the array were referenced at an interferometer for subtraction of system generated noise, and attenuated before the demultiplexer to achieve a peak optical power of $27 \mathrm{dBm}$ per wavelength at the receiver as required to obtain the desired shot noise limited performance. The demultiplexer was used to drop the signal bearing channels. The output of the demultiplexer was then detected and demodulated to extract the phase information.

\section{EXPERIMENT RESULTS}

To measure the system phase sensitivity, a sinewave signal at $1 \mathrm{kHz}$ with an amplitude equivalent to $-63 \mathrm{~dB}$ re $1 \mathrm{rad}$ in radians was applied on the PZT at the Michelson sensor in the "TDM group". The phase spectrum was tested under a wide range ( 29 to $59 \mathrm{~dB}$ ) of TDM group insertion loss (IL). The IL of one sensor was assumed to be $5 \mathrm{~dB}$, increasing by $6 \mathrm{~dB}$ for every doubling of the number of sensors in the TDM group, as is consistent with current sensor technology. The optical spectrum at the output of the array was characterized for a TDM group IL of $32 \mathrm{~dB}$, as illustrated in Figure 3 (a). It can be seen from the spectrum that the optical signal to noise ratio (OSNR) for each channel is better than $35 \mathrm{~dB}$, which implies that the noise added by the distributed EDFA is sufficiently low that it can effectively be ignored.

Figure 3 (b) shows the demodulated phase spectra range from $100 \mathrm{~Hz}$ to $2 \mathrm{kHz}$ interrogated at the last channel (1545.32 $\mathrm{nm}$ ) with two TDM group ILs of $32 \mathrm{~dB}$ and $43 \mathrm{~dB}$. The IL of the two illustrated spectra in the figure is close to the loss of a TDM group with 16 and 64 sensors addressed by a single wavelength respectively. This equates to a total number of 256 and 1024 sensors respectively. It can be seen from the figure that the system phase noise floor remains almost the same with increasing effective number of sensors ranging from 16 to 64 per wavelength, achieving a phase resolution of around $-90 \mathrm{~dB}$ re $1 \mathrm{rad} / \mathrm{Hz}^{1 / 2}$. The system phase noise floor shows a flat spectrum from 100 to $2000 \mathrm{~Hz}$ and thus we define the phase resolution in the sensor system as the mean value of the system phase noise floor from $300 \mathrm{to} 800 \mathrm{~Hz}$, and the best system resolution is calibrated to be $-90 \mathrm{~dB}$ re $1 \mathrm{rad} / \mathrm{Hz}^{1 / 2}$ in a passive system limited only by the laser noise. (below $100 \mathrm{~Hz}$ noise is limited by environmental noise).

The table above the spectra demonstrates the relationship between the number of sensors addressed by a single wavelength, the total number of sensors with 16 wavelengths, IL of the TDM group, and the predicted phase resolution with a practical sampling rate and pulse-width. It can be seen from the table that the phase noise floor is increased to -77 $\mathrm{dB}$ re $1 \mathrm{rad} / \mathrm{Hz}^{1 / 2}$ when the number of sensors is increased to 256 per wavelength. This can be explained as follows. Due to the amplification employed in the array, the signal-amplified spontaneous emission (ASE) beat noise contributes to the system noise floor. In the small-signal regime, due to the high population inversion a low noise figure is obtained and the generated ASE noise can be ignored. However, as the IL of the TDM group is increased, a higher gain is required and a lower input signal is injected at the EDFA input. ASE builds up rapidly and starts to help saturate the EDFA giving rise to signal-ASE beat noise which ultimately becomes the dominant noise source and increases the system noise floor as the number of sensors per wavelength is increased. On the other hand, TDM architectures inherently sample each sensor at the interrogation repetition rate. For a given pulse-width, the phase noise observed on each sensor increases with an increasing number of sensors in the TDM group, because the bandwidth available for each sensor decreases. 


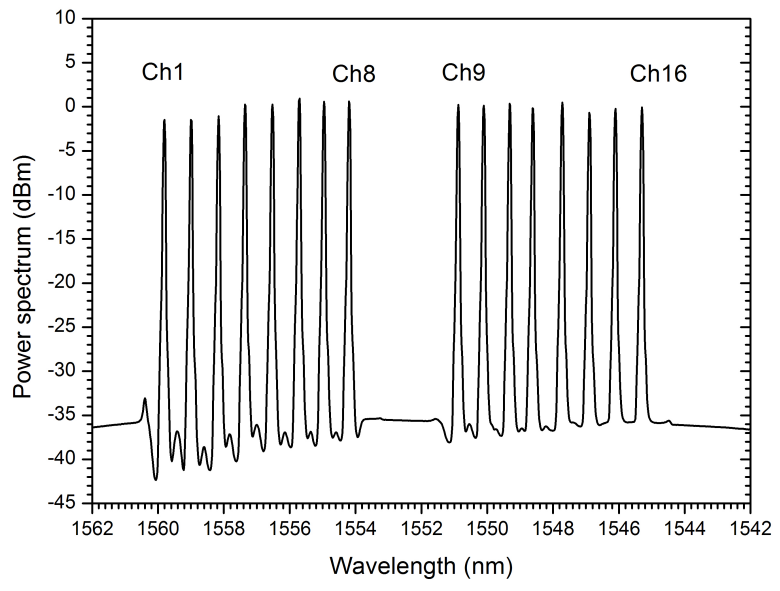

(a)

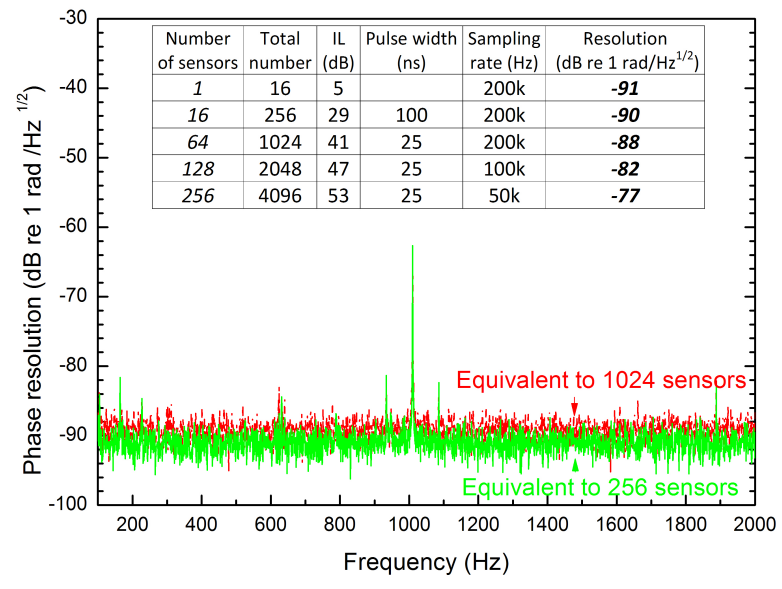

(b)

Figure 3 (a) Optical output spectrum from the array with the TDM group insertion loss equivalent to 256 sensors. The OSNR is larger than $35 \mathrm{~dB}$ at each channel. (b) Demodulated phase spectra of the array output with TDM group insertion losses equivalent to 256 and 1024 sensors with 16 wavelengths. The peak corresponds to the ac modulation of the Michelson sensor. The table illustrates the relationship between the predicted phase resolution and the number of sensors per wavelength.

Our results show that the experimental arrangement can address up to $64 \times 16$ sensors with a phase resolution limited only by the noise of our lasers and can support up to $256 \times 16$ with a perfectly acceptable phase resolution for many applications of $-77 \mathrm{~dB}$ re $1 \mathrm{rad} / \mathrm{Hz}^{1 / 2}$ even with an adjusted sampling rate. It is to be appreciated that increasing the number of sensors per wavelength means decreasing the duty cycle in the time domain. Bandwidth limitations and added aliased high frequency noise in the TDM architecture ultimately limit the highest level of multiplexing possible, even with a suitably adjusted sampling rate.

\section{CONCLUSION}

In conclusion, we have demonstrated what we believe to be the first experiment addressing acoustic sensing in a distributed amplified array based on a hybrid TDM and DWDM architecture. Our experimental results show that the array is able to support a total number of 1024 sensors along one interlink fibre pair, allowing a phase resolution of around $-90 \mathrm{~dB}$ re $1 \mathrm{rad} / \mathrm{Hz}^{1 / 2}$, limited only by laser noise. Further, we show that the current 16 -wavelength array could theoretically be expanded to interrogate 4096 sensors, albeit with a slight compromise in phase resolution. The number of interrogation sensors could be further increased by increasing the number of wavelength channels. We consider that this architecture represents the best approach to support a distributed interferometric sensing array of more than 1000 sensors through one pair of interlink fibres, using only commercially available components.

\section{REFERENCES}

[1] C. K. Kirkendall, and A. Dandridge, "Overview of high performance fibre-optic sensing", Journal of Physics D: Applied Physics, 37(18), R197 (2004).

[2] G. A. Cranch, and P. J. Nash, "Large-scale multiplexing of interferometric fiber-optic sensors using TDM and DWDM", Journal of Lightwave Technology, 19(5), 687-699 (2001).

[3] A. C. Geoffrey, and J. N. Phillip, "High multiplexing gain using TDM and WDM in interferometric sensor arrays", SPIE 3860, 531-537 (1999).

[4] C. W. Hodgson, M. J. F. Digonnet, and H. J. Shaw, "Large-scale interferometric fiber sensor arrays with multiple optical amplifiers", Optical Letters, 22(21), 1651-1653 (1997).

[5] C. W. Hodgson, J. L. Wagener, M. J. F. Digonnet et al., "Optimization of large-scale fiber sensor arrays incorporating multiple optical amplifiers.", Journal of Lightwave Technology, 16(2), 218-231 (1998).

[6] P. Nash, and A. Strudley, "High efficiency TDM/WDM architectures for seismic reservior monitoring", 20th International Conference on Optical Fiber Sensors 7503, T1-T4 (2009). 